\title{
The principle curvature-driven diffusion model for image de-noising
}

\author{
Qiao Xin ${ }^{1}$
}

\begin{abstract}
In this paper, we discuss a weighted average of the maximum and minimal principle curvature as the diffusion coefficient for de-noising digital images with an additive noise. The main advantage of this approach is that it preserves important structures similar to Gauss curvature-driven diffusion, and it has a stable and fast numerical algorithm. Moreover, the proposed model helps gaining better de-noising results than Gauss curvature-driven diffusion.
\end{abstract}

Keywords Image de-noising $\square$ Perona and Malik equation $\square$ Principle curvature $\square$ Gauss curvature $\square$ Diffusion coefficient

\section{Introduction}

Image de-noising is an important preprocessing step for many applications, such as image segmentation, image inpainting, pattern recognition, and robot vision and so on. We consider the simplest image degradation model:

$$
u_{0}(x, y)=u(x, y)+n(x, y)
$$

where $u_{0}(x, y)$ is the degrade image or observed image, $u(x, y)$ is the real scene and $n(x, y)$ is the additive Gaussian white noise. We want to recover $u(x, y)$ knowing $u_{0}(x, y)$. Different methods such as stochastic modelling, wavelets, and PDE methods, have been proposed to solve this problem, the excellent introduction of the relevant work can be found in [1].

In this paper, we consider a much popular PDE method. Because of the work of Perona and Malik[2], who point out that non-linear diffusion filters can preserve the important image edges while removing noises, the PDE methods are now widely used in image de-noising. In Perona and Malik's model, the grey levels of image $u(x, y)$ are diffused according to:

\footnotetext{
${ }^{1}$ Q. Xin $(\varangle)$

College of Mathemactics and Statistics, Yili Normal University, Yining, China

e-mail:xinqiaoylsy@163.com
} 


$$
\partial u / \partial t=d \dot{\boldsymbol{v}}(c(x, y, t) \nabla u),
$$

where $c(x, y, t)=s^{2} /\left(s^{2}+|\nabla u|^{2}\right)$, called diffusion coefficient, $s$ is a threshold. More information about this model can be found in [2]. Note that if we choose $c(x, y, t)=1$, then equation (1.2) becomes the standard linear heat equation or the classical low-pass filter [3, pp.95-96]. In general, the diffusion coefficient $c(x, y, t)$ can be chosen as a non-increasing function of the gradient [4-8]. From their research, we know that the diffusion coefficient $c(x, y, t)$ governs the behaviour of the diffusion process. Thus, how to choose a better suitable diffusion coefficient becomes an interesting and important factor in using PDE to treat image de-noising.

\section{The proposed model}

In the last decade, the intensity images are treated as Monge surfaces in image processing [9-14]. The parameterization of the Monge surface of the intensity image is given by

$$
I(x, y)=(x, y, u(x, y))
$$

We can find the principle curvatures of the image surface $I(x, y)$ as follows:

$$
k_{1}=H+\sqrt{H^{2}-K} \text { and } k_{2}=H-\sqrt{H^{2}-K}
$$

where $H$ and $K$ are the mean and Gauss curvature of the image surface.

Thus, the geometric property of the image surface can be introduced in image denoising. As shown in [15], they propose a model based on mean curvature for image inpainting(CCD) by choosing $c(x, y, t)=H$ as the control term. The authors in [16] propose a Gauss curvature-driven diffusion (GCDD) by assuming $c(x, y, t)=\phi(|K|)$ where $\varphi: R^{+} \cup 0 \rightarrow \mathrm{R}^{+} \cup 0$ is a nonnegative valued function with $\phi(0)=0$, and the authors also point out that the de-noising scheme can preserve important image structures, such as straight edges, curvy edges, ramps, corners, small-scaled features, etc.

In the first experiment described below, we want to show that $|K|$ as the diffusion coefficient is not suitable for some artificial images (For example Figure 1, (a)). In the experiment, we use the central difference techniques to calculate the value of $K$ and $H$. Apply formulas (2.2), we find the value of $k_{1}$ and $k_{2}$. For the $100^{\text {th }}$ row of the image (Figure 1, (a)), the results of our experiment are summarized as in Figure 1, (b). The value of Gauss curvature is equal to 160 (See Figure 1 (b), Top Right) which is much larger than the value of principal curvatures which are equal to 13 (See Figure 1 (b), Button) on the image edge. So, based on 
our experiments on a comparison the value of $K$ with $k_{1}$ and $k_{2}$, we conclude that the GCDD model blur the image edge more serious than the principal curvature when used as the diffusion coefficient in equation (1.2). Hence, it seems that the principal curvature as the diffusion coefficient in equation (1.2) is more suitable than Gaussian curvature in the area of edge preserved.

At first, define $k_{\min }=\min \left\{\left|k_{1}\right|,\left|k_{2}\right|\right\}$ and $k_{\max }=\max \left\{\left|k_{1}\right|,\left|k_{2}\right|\right\}$. Next, we want to investigate the de-noising or edge preserved ability of $k_{\min }$ and $k_{\max }$ as the diffusion coefficient in equation (1.2), respectively. In fact, the minimal principal curvature, $k_{\min }$ along the edge is zero, and the noise has a large value of maximal principal curvature $k_{\max }$ [16]. In the second experiment, we choose $k_{\min }$ and $k_{\max }$ as the diffusion coefficient, which is called minimal and maximal principal curvature-driven diffusion, respectively. We use the GCCD's numerical algorithm to solve the minimal and maximal principal curvature-driven diffusion model. The denoising results of the minimal and maximal principal curvature-driven diffusion are summarized as in Figure 2 (c) and (d). Based on our experiments for a binary image, we get the following results, the edge can be better preserved by the minimal principal curvature-driven diffusion with long time (See Figure 2(c)) and the white Gaussian noise is effectively removed by the maximal principal curvaturedriven diffusion (See Figure 2 (d)). So the $k_{\min }$ and $k_{\max }$ as the diffusion coefficient, respectively, which produce different effect in image de-noising.

Through the previous analysis, in this paper, we propose another choice of the diffusion coefficient, which is based on the weighted average of the maximal and minimal principal curvature. This new model can combine with the advantages of two diffusion coefficients, i.e. edge preserved and noise efficiently removed. This new model also preserves important geometric structures of image similar to GCDD, and it has a fast and stable numerical algorithm. The new model is described in Section 2. The numerical implementation is discussed in Section 3, and then we compare the de-noising results with GCDD in various natural images.

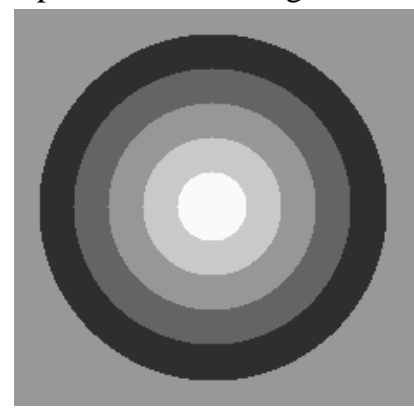

(a)
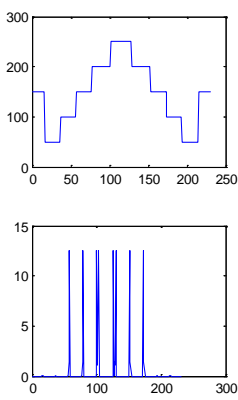

(b)
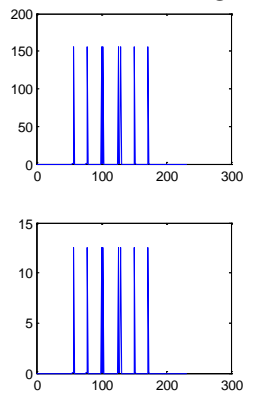

Fig. 1 (a) Original image (b) Top Left: the value of original image (Row 100), Top Right: the value of Gaussian curvature of original (Row 100), Button Left: the minimal principal curvature value of original image (Row 100), Button right: the maximal principal curvature value of original image (Row 100). 


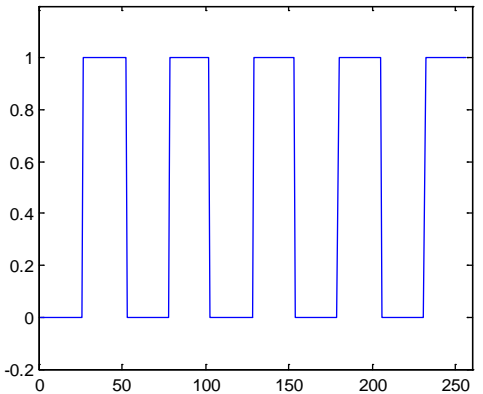

(a)

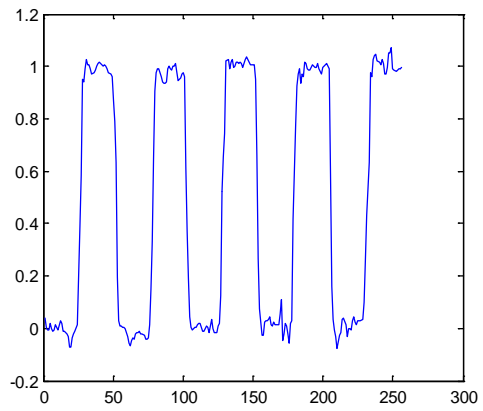

(c)

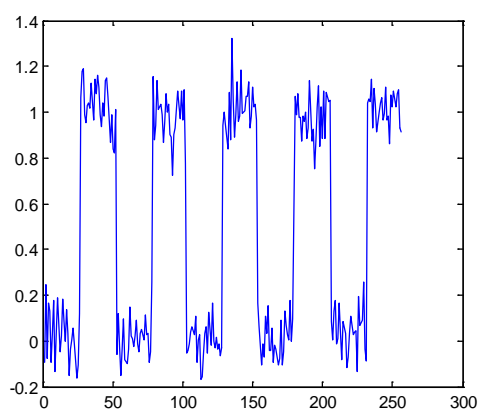

(b)

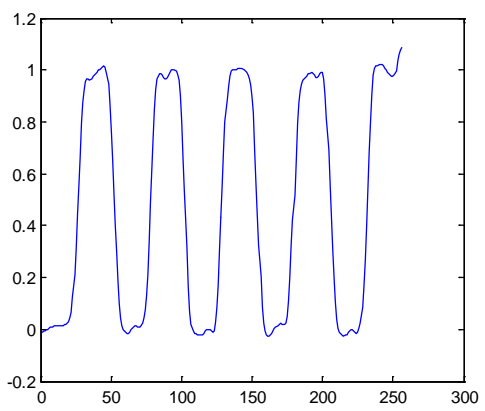

(d)

Fig. 2 Experiments on binary image (a) Exact image (b) Noised exact image (c) Noise removed by the minimum curvature-driven diffusion $(/=1)$ (d) Noise removed by the maximal curvature-driven diffusion $(I=0)$.

In GCDD model, choosing $\phi(|K|)=\sqrt{\left|k_{1} k_{2}\right|}$ as the diffusion coefficient, then the diffusion coefficient is the geometric average of the absolute value of the minimal and maximal principle curvatures. Moreover, in the CDD image inpainting model, the control term is chosen as $H=\left(k_{1}+k_{2}\right) / 2$, then it is the arithmetic average of the minimal and maximal principle curvatures. Motivated by this, we consider instead of the diffusion coefficient $c(x, y, t)=\phi(|K|)$ in GCDD by the weighted average of the principal curvatures, and then we propose the following parabolic PDE with Neumann boundary condition:

$$
\begin{cases}u_{t}(x, y, t)=d \dot{v}\left(\varphi\left(k_{1}, k_{2}\right) \nabla u\right), & (x, y) \in \Omega, t>0, \\ \partial u / \partial n=0, & (x, y) \in \partial \Omega, t>0, \\ u\left(x, y, 0 \neq u_{0}(x, y),\right. & (x, y) \in \Omega,\end{cases}
$$

where $\varphi\left(k_{1}, k_{2}\right)=\arctan \left((1-\lambda(t)) k_{\min }+\lambda(t) k_{\text {max }}\right), k_{1}, k_{2}$ is the principal curvature of image surface $I(x, y)=(x, y, u(x, y)), 0<\lambda(t)<1$ is positive weighted parameter, $\Omega$ denotes the entire domain of the image, $n$ is outer normal direction to 
$\partial \Omega$. Note that if we let $\varphi\left(k_{1}, k_{2}\right)=(1-\lambda) k_{\min }+\lambda k_{\max }$, thus if $\lambda \equiv 0$, the equation (2.3) is the minimal principal curvature-driven diffusion, and if $\lambda \equiv 1$, the equation (2.3) is the maximal principal curvature-driven diffusion. The aim of the choice of weighted mean of the principal curvature as the diffusion control term is to combine the advantages of the two diffusion coefficients.

Now, we analyze the choices of parameter $\lambda(t)$ and the function $\phi$. We note that the smaller diffusion coefficient stands for slow diffusion, however, the larger corresponding stands for fast diffusion. Thus, if we want to preserve the edge while removal the noise, we need the Equation (2.1) is a slow diffusion on the edge and texture, the equation (2.3) corresponding is a fast diffusion on noise. Since the minimal principal curvature $k_{\min }$ along the edge is zero, and the noise has a large value of maximal principal curvature $k_{\max }$. So we need $\lambda(t) \rightarrow 0$ along the edge, and then the equation (2.3) is similar to the minimal principal curvaturedriven diffusion on the edge and texture, it is also the slow diffusion. In the following, we give a simple way to set the parameter $\lambda(t)$. To do this, we use the following inequality:

$$
\sqrt{\left|k_{1}\right|^{2}+\left|k_{2}\right|^{2}} \geq k_{\text {max }} \geq\left(\left|k_{1}\right|+\left|k_{2}\right|\right) / 2 \geq \sqrt{\left|k_{1} k_{2}\right|} \geq k_{\text {min }} .
$$

Note that the bigger diffusion coefficient can smooth the noise and while blur the edge, and then for our aim of edge preserved, since the GCDD model can better preserved the edge, so we need

$$
(1-\lambda(t)) k_{\min }+\lambda(t) k_{\max } \leq \sqrt{\left|k_{1} k_{2}\right|},
$$

thus, the new model also can better preserve the edge. By solving the above inequality, we get the range of $\lambda(t)$ on the edge as follows:

$$
0 \leq \lambda(t) \leq \sqrt{k_{\min }} /\left(\sqrt{k_{\min }}+\sqrt{k_{\max }}\right)
$$

In the experiment, the parameter $\lambda(t)=0.1 /(K t+1)$ which is dependent on the time variable, where $K$ is a constant, which determines the rate of decrease. At the beginning of the diffusion process, our proposed model can efficiently remove the noise, with the increase of the iterations, the parameter $\lambda(t) \rightarrow 0$, and then, the value of $\lambda(t)$ will be satisfied the inequality (2.4), thus, the edge can be preserved by our proposed. Next, for the choice of function of $\varphi(\cdot)=\arctan (\cdot)$, the aim is to keep the numerical algorithm stable (The details see below)and the stable condition is $\Delta t \leq 1 / 2 \pi$ (see Theorem 3.1). Of course, the function $\varphi(\cdot)$ has many other choices, which should be chosen as the monotone increasing bounded function. 


\section{Numerical Algorithm and Experimental Results}

The details of our numerical algorithm are as follows. First, the tested images are represented by matrices of intensity values, where each matrix element $u_{i, j}$ is a real value correspondent to the gray scale level of the image $u(x, y)$ at the point $x=x_{i}=i \Delta x$ and $y=y_{j}=j \Delta y$, as usual, we suppose $\Delta x=\Delta y=h$. We denote $u\left(x_{i}, y_{j}, t_{n}\right)$ by $u_{i, j}^{n}$, where $t_{n}=n \Delta t$, and $\Delta t$ is the time scale. The derivative of $u$ in relation to the time $t$ (space scale parameter), that is, $u_{t}$ calculated in $\left(x_{i}, y_{j}, t_{n}\right)$ is approximated by Euler's method. For the approximation of the principal curvature $k_{1}$ and $k_{2}$, we use the central difference techniques. We refer to [7].

The explicit scheme of Equations (2.1) iterates as

$$
u^{n+1}=u^{n}+\Delta t\left(\nabla\left(\varphi\left(k_{1}, k_{2}\right) \nabla u\right)\right),
$$

and $\varphi\left(k_{1}, k_{2}\right)$ denotes the conductance function based on the principal curvature at the given point. We use a scheme which combines forward and backward differences for the discretization of the differentiation. Then, $\nabla\left(\varphi\left(k_{1}, k_{2}\right) \nabla u\right)$ can be discretized as follows:

$$
\begin{aligned}
& \nabla\left(\varphi\left(k_{1}, k_{2}\right) \nabla u\right)= \\
& \frac{1}{h^{2}}\left(\varphi\left(k_{1 ; i+1 / 2, j}, k_{2 ; i+1 / 2, j}\right)\left(u_{i+1, j}-u_{i, j}\right)+\varphi\left(k_{1 ; i-1 / 2, j}, k_{2 ; i-1 / 2, j}\right)\left(u_{i-1, j}-u_{i, j}\right)\right. \\
& \left.+\varphi\left(k_{1 ; i, j+1 / 2}, k_{2 ; i, j+1 / 2}\right)\left(u_{i, j+1}-u_{i, j}\right)+\varphi\left(k_{1 ; i 2, j-1 / 2}, k_{2 ; i, j-1 / 2}\right)\left(u_{i, j-1}-u_{i, j}\right)\right),
\end{aligned}
$$

where $k_{1 ; i \pm 1 / 2, j \pm 1 / 2}, k_{2 ; i \pm 1 / 2, j \pm 1 / 2}$ is the value of $k_{1}, k_{2}$ at location $(i \pm 1 / 2, j \pm 1 / 2)$, which can be obtained by interpolation, then using Neumann's boundary conditions and formula (3.1) we calculate $u_{i, j}^{n}$ for $n=1,2, \cdots, N$.

Since the diffusion equations backwards in time can give rise to numerically unstable computational methods, unless the problem is appropriately constrained or reformulated [17]. In fact, the numerical scheme (3.1) is conditionally stable.

Theorem 3.1. Assume $h=1$ and $\Delta t \in(0,1 / 2 \pi)$, and then, we have the numerical scheme (3.4) is conditionally stable with respect to the sup norm.

Now, we investigate the de-noised ability of the proposed model for nature image and compare the experiment results with GCDD model. The experiments on Lena.bmp and Goldhill.bmp as followed. We choose the parameter $K=0.8$, $\Delta t=0.1$ and the noisy variance $\sigma=20$ in all our experiments. In fact, the small parameter $\lambda$ can help to decrease the iteration numbers, and also reduce the computational effort. Thus, in the experiments, as usual, the number of iterations of our method is 100, but the number of iterations of GCDD model equals to 300 . 
For GCDD model, we want to keep the stability of the scheme, we also choose $\phi(\cdot)=\arctan (|K|)$. From the Figure 3,4 we demonstrate that the proposed model can obtain better de-noising results than GCDD model in the area of SNR.

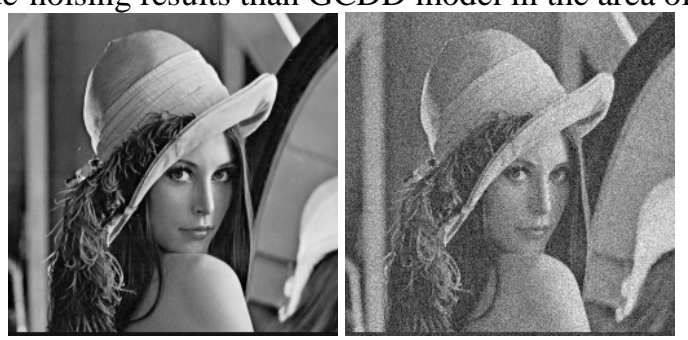

Lena.bmp

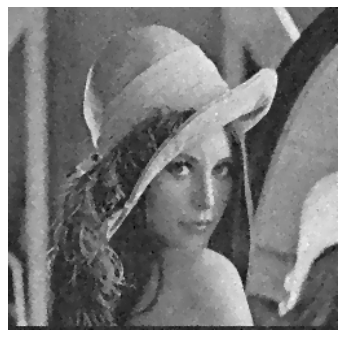

(b) (a)

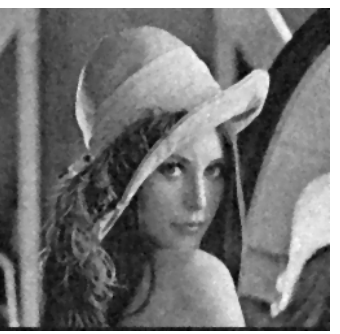

(c)

Fig. 3 Experiments on Lena.bmp. (a) Noisy image(SNR= 8.4800) (b) GCDD model for noise removal(SNR= 13.9166) (c) The proposed model for noisy removal(SNR=14.8340).

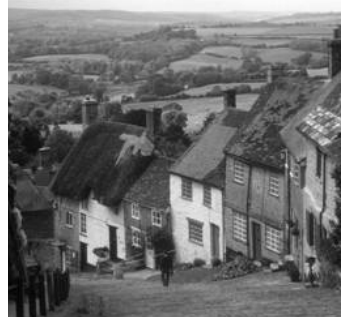

Goldhill.bmp

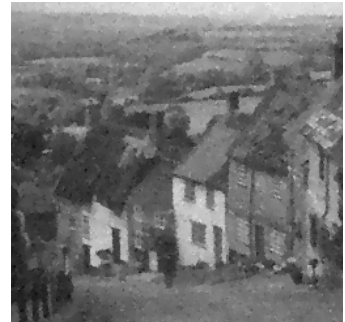

(b)

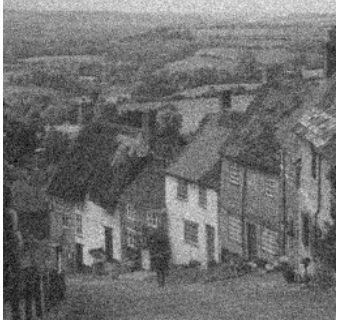

(a)

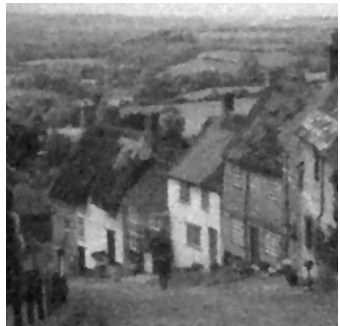

(c)

Fig. 4 Experiments on goldhill.bmp (a) Noisy image(SNR=7.7599) (b) GCDD model for noise removal(SNR=13.0787) (c) The proposed model for noisy removal(SNR=14.3114). 
Acknowledgments This study is funded by supported by the general project of Yili Normal University (No. 2012YB016).

\section{References}

1. Liu C, Szeliski R, Kang S B et al (2008) Automatic estimation and removal of noise from a single image. IEEE Trans Pattern Anal Mach Intell 30:299-314.

2. Perona P, Malik J (1990) Scale-space and edge detection using anisotropic diffusion. IEEE Trans Pattern Anal Mach Intell 12:629-639.

3. Aubert G, Kornprobst P (2006) Mathematical Problems in Image Processing: Partial Differential Equations and the Calculus of Variations. Springer, New York.

4. Rudin L, Osher S, Fatemi E (1992) Nonlinear total variation based noise removal algorithms. Physica D 60:259-268.

5. Chambolle A, Lions P L (1997) Image recovery via total variation minimization and related problems. Numer Math 76:167-188.

6. Blomgren P, Chan T F, Mulet P et al (2000) Variational PDE models and methods for image processing, in: Griffiths D F, Watson G A (Eds.), Numerical analysis, Chapman \& Hall/CRC, Florida.

7. Chen Y, Levine S, Rao M (2006) Variable exponent, linear growth functionals in image restoration. SIAM J Appl Math 66:1383-1406.

8. Bollt E M, Chartrand R, Esedoglu S et al (2009) Graduated adaptive image denoising: local compromise between total variation and isotropic diffusion. Adv Comput Math 31:61-85.

9. A.I.EI-Fallah, G.E.Ford, On mean curvature diffusion in nonlinear image filtering, Pattern Recognit. Lett. 19(1998)433-437.

10. Sochen N, Kimmel R, Malladi R (1998) A geometrical framework for low level vision. IEEE Trans Image Process, Special Issue on PDE based Image Processing 7:310-318

11. Yezzi A (1998) Modified curvature motion for image smoothing and enhancement. IEEE Trans Image Process, Special Issue on PDE based Image Processing 7:345-352.

12. Malladi R, Sethian J A (1996) Image processing: Flows under Min/Max Curvature and Mean Curvature. Graphical Models and Image Processing 58:127-141.

13. Mota C, Gomes J (1999) Curvature operators in geometric image processing, In Brazilian Symposium on Computer Graphics and Image Processing, IEEE Press.

14. Barth E, Caelli T, Zetzsche C (1993) Image encoding, labeling, and reconstruction from differential geometry. Graphical Models and Image Processing 55:428-446.

15. Chan T F, Kang S, Shen J (2002) Euler's elastica and curvature-based image inpainting. SIAM J Appl Math 63:564-592

16. Lee S H, Seo J K (2005) Noise Removal with gauss curvature-Driven diffusion. IEEE Trans Image Process 14:904-909.

17. Hummel A, Kimia B, Zucker S (1987) Deblurring Gaussian blur. Comput Vision Graphics Image Processing 38:66-80. 